\title{
Analysis of the Interaction between Tropomyosin Allergens and Antibodies Using a Biosensor Based on Imaging Ellipsometry
}

\author{
Wei Wang, ${ }^{\dagger, \dagger}$ Cai Qi, ${ }^{\dagger, \perp}$ Teng-fei Kang," Yu Niu," Gang Jin," Yi-qiang Ge, ${ }^{*,+\S}$ and Ying Chen ${ }^{* \dagger}$ \\ ${ }^{\dagger}$ Agro-product Safety Research Center, Chinese Academy of Inspection and Quarantine, \#3, Gaobeidian North Rd., Beijing 100123, \\ China \\ ${ }^{\ddagger}$ College of Food Science \& Nutritional Engineering, China Agricultural University, Beijing 100083, China \\ ${ }^{\S}$ China Rural Technology Development Center, Beijing 100045, China \\ "Institute of Mechanics, Chinese Academy of Sciences, \#15, Beisihuan West Rd., Beijing 100190, China \\ ${ }^{\perp}$ Institute of Equipment Technology, Chinese Academy of Inspection and Quarantine, \#3, Gaobeidian North Rd., Beijing 100123, \\ China
}

ABSTRACT: A biosensor based on high spatial resolution imaging ellipsometry has been studied to examine its role in the rapid detection and analysis of the tropomyosin allergen existing in crustaceans. This methodology has been established for detection of the tropomyosin allergen and includes ligand screening, the determination of sensitivity, and a comparison with traditional detection methods. Three kinds of monoclonal antibodies (2F9, 4C7, and 2H6) known to have a high bioactivity against the tropomyosin allergen were screened and separately immobilized as ligands on a silicon wafer surface, thus allowing them to capture the tropomyosin allergen. Resulting changes on the wafer surface were visualized in gray scale variation on an ellipsometry image. Images showed that

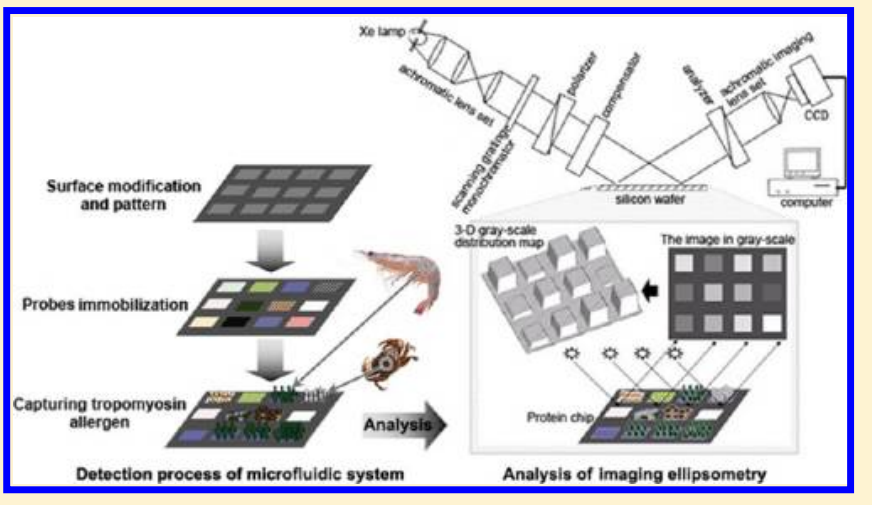
these antibodies are able to recognize the presence of the tropomyosin allergen in shrimp and crab with sensitivity of $1 \mathrm{mg} \mathrm{L}^{-1}$, and at a detecting time of approximately $30 \mathrm{~min}$ for an extracted sample. This preliminary study has shown that the detection of the tropomyosin allergen is rapid and specific when using this type of assay on products containing shrimp and crab. When compared with the enzyme-linked immunosorbent assay, the biosensor based on imaging ellipsometry is able to perform a fast label-free analysis.

$\mathrm{F}$ ood allergy is a serious health concern affecting approximately five to eight percent of young children and about two to four percent of adults. ${ }^{1,2}$ The severity of food allergic reactions varies from mild urticaria to life-threatening anaphylactic shock and asthma. The top eight common allergenic foods are milk, crustaceans, eggs, fish, tree nuts, peanuts, soybean, and wheat. ${ }^{3}$ Crustaceans (such as shrimps and crabs) represent one of the most common causes of food allergies and are frequently linked to anaphylaxis or hypersensitivity. The only major heat-resistant allergen of crustaceans has been identified as the $34-36 \mathrm{kDa}$ muscle protein tropomyosin (TM). ${ }^{4,5}$ Thus, appropriate analytical techniques are necessary to ensure the rapid and sensitive identification of this crustacean allergen and to ensure the surveillance of labeling requirements by the responsible authorities. Currently, most existing research has been dedicated to the identification and characterization of allergens from a crustacean origin, ${ }^{6-9}$ with only a few methods described specifically for the detection of crustaceans or other allergens. ${ }^{10-13}$ These existing methods, however, have shortcomings such as the need for tracer labeling, an indirect format of detection, and overall time consumption. Thus, there is an urgent need for a method of detection proven to be more rapid, simple, and direct.

A biosensor based on imaging ellipsometry (BIE) was developed in 1995. The BIE combines high spatial resolution imaging ellipsometry with a microfluidic system to analyze macromolecular interactions. ${ }^{14-17}$ Imaging ellipsometry records the light reflection intensity $(I)$ and represents layer thickness (d) of the ultrathin films within protein chips. ${ }^{16}$ Supposing that the refractive index is invariant, the relationship between light intensity $(I)$ and layer thickness $(d)$ in the range of $0-30 \mathrm{~nm}$ can be expressed as $I=k \times d .{ }^{18}$ Under the same conditions for protein and ellipsometry, " $k$ " is constant and can be determined from a protein layer with known light intensity and thickness. ${ }^{19}$ On the other hand, there is a relation between the surface concentration and film thickness: surface concentration $(\mu \mathrm{g}$ $\left.\mathrm{cm}^{-2}\right) \approx K \times d$, where $K=0.12 .{ }^{20}$ When proteins accumulate on the surface of a protein chip, whether by absorption or

Received: December 27, 2012

Accepted: March 21, 2013

Published: March 21, 2013 
interaction, they will effectively change the layer thickness of the chip. As such, this change of layer thickness can be used to characterize macromolecular interactions. Thus far, BIE has been successfully applied to numerous biomedical fields including a clinical diagnosis for hepatitis $\mathrm{B}^{21}$ cancer marker tests, $^{22,23}$ bacterium and virus detection, ${ }^{24-26}$ screening and identification of antibodies against severe acute respiratory syndrome (SARS), ${ }^{27}$ and biomolecule interactions. ${ }^{28}$ These applications have demonstrated that the BIE method has allowed the development of a quantitative, label-free, rapid, high-throughput, and intuitionistic image while maintaining a low-cost analysis of biological interactions.

In this study, we report on a BIE detection procedure developed specifically for the TM allergen. Three monoclonal antibodies (Mc Abs) were screened against the TM allergen and then immobilized covalently as ligands or bioprobes on the surface of silicon wafers. ${ }^{16,29}$ The specificity of Mc Abs and the sensitivity of BIE in detecting the TM allergen were then determined. The standard curve representing different concentration gradients was computed to assess the quantitative detection of the TM allergen. As such, the TM allergen levels in commercial food products were confirmed by collecting BIE measurements and then compared using the enzyme-linked immunosorbent assay (ELISA) test method.

\section{EXPERIMENTAL SECTION}

Materials and Reagents. A study on the TM standard sample and three specific Mc Abs known to have resistance against TM (2F9, 4C7, and 2H6 from Mouse IgG1) was granted by the Medical College at Shenzhen University. ${ }^{30,31}$ ELISA kits including Shellfish Assay and Fast Crustacean Kits were purchased from Neogen (USA) and R-Biopharm (Germany), respectively. Silicon wafers were purchased from the General Research Institute for Nonferrous Metals (China). Components of the study including Tween 20, N-hydroxysuccinimide (NHS) and 1-(3-dimethyla-minopropyl)-3ethylcarbodiimide hydrochloride (EDC), bovine serum albumin (BSA), protein A, human immunoglobulin $G(\operatorname{IgG})$, and Glycine (Gly) were produced by Sigma. Phosphate-buffered saline solution (PBS, $140 \mathrm{mM} \mathrm{NaCl}, 2.7 \mathrm{mM} \mathrm{KCl}, 10 \mathrm{mM}$ $\mathrm{Na}_{2} \mathrm{HPO}_{4}$, and $1.8 \mathrm{mM} \mathrm{KH}_{2} \mathrm{PO}_{4}, \mathrm{pH} 7.3$ ) with $1 \%$ Tween 20 (PBST) was prepared in deionized water $(18.3 \mathrm{M} \Omega \mathrm{cm}$, a MilliQ plus system from Millipore, Bedford, MA). Commercial food products including fresh live shrimp and crab, shrimp crackers, shrimp paste, shrimp meat balls, and shrimp strips in addition to negative samples (consisting of powdered milk, soy, eggs, fish, and graham bread) were purchased from a local supermarket in Beijing, China.

Antibodies against the TM allergen were diluted to a concentration of $0.1 \mathrm{mg} \mathrm{mL}^{-1}$ with PBST. Ingredients of the blocking reagents were combined at a mixture of $10 \mathrm{mg} \mathrm{mL}^{-1}$ BSA and $10 \mathrm{mg} \mathrm{mL}^{-1}$ Gly. Crude TM allergen extracts obtained from commercial food products were obtained by the extraction method described by Qiao, and only minor modifications to this method were taken. ${ }^{32}$ Thirty grams of commercial food products were homogenized with $30 \mathrm{~mL}$ of egg white in $100 \mathrm{~mL}$ of PBS. The mixture was stirred gently overnight at a temperature of $4{ }^{\circ} \mathrm{C}$ and then centrifuged for 15 $\min$ at $4000 \mathrm{rpm}$. The supernatant was then transferred to a new tube and secondarily centrifuged for a $15 \mathrm{~min}$ period at $10000 \mathrm{rpm}$. Finally, the supernatant was stored at $-20{ }^{\circ} \mathrm{C}$ for further use. Proteins in the negative samples were extracted following the same process.
TM Allergen Detection Using BIE. Surface Modification.

Silicon wafers were selected as the substrate for analysis and cut into individual element sizes of $20 \mathrm{~mm} \times 15 \mathrm{~mm}$. The carboxyl modification method was used to immobilize antibodies against the TM allergen. ${ }^{21}$ When a modified silicon wafer was placed into the lab-built microfluidic system, ${ }^{17,21}$ the carboxyl groups situated on its surface were activated by NE (consisting of 0.05 $\mathrm{M}$ NHS and 0.2 M EDC in deionized water at a concentration of 10 micro liters $(\mu \mathrm{L})$ per unit and passed through at a concentration of a $\left.10 \mu \mathrm{L} \mathrm{min}{ }^{-1}\right)$. NE was then able to transfer carboxyl groups into the sulfo-NHS ester, where they were able to react with the $-\mathrm{NH}_{2}$ groups of protein to immobilize antibodies covalently as ligands.

Ligand Immobilization. During the next step, the modified wafers were placed into a microfluidic system of BIE. This allowed the wafer surfaces to be patterned in an array format as regular small cells, with the elemental size of each cell being about $1.5 \mathrm{~mm} \times 1 \mathrm{~mm}$. Antibodies against the TM allergen types $2 \mathrm{~F} 9,2 \mathrm{H} 6$, and $4 \mathrm{C} 7$ were then pumped into each cell and immobilized separately as ligands in the microarray $(10 \mu \mathrm{L}$ per

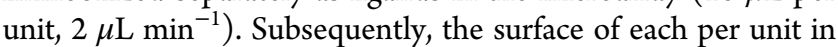
microarray was blocked and optimized with BSA and Gly block reagents $\left(40 \mu \mathrm{L}\right.$ per unit, $\left.2 \mu \mathrm{L} \mathrm{min}^{-1}\right)$. The units were rinsed

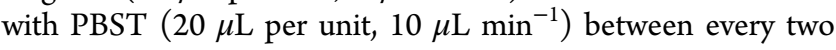
consecutive operation steps. As such, a multiplex microarray containing a sensing surface array was formed for further use.

Detection of TM Allergen. After blocking, the TM allergen protein or shrimp samples were brought into contact with the immobilized ligands on the sensing surface. First, the shrimp TM allergen protein standard sample was added $\left(0.1 \mathrm{mg} \mathrm{mL}^{-1}\right.$, $10 \mu \mathrm{L}$ per unit, $2 \mu \mathrm{L} \mathrm{min}^{-1}$ ) with the allergen captured from the specific ligands passing over the sensing surface. Subsequently, the wafer surface was rinsed with deionized water $(25 \mu \mathrm{L}$ per unit, $10 \mu \mathrm{L} \mathrm{min}-1$ ), and the microarray was removed from the microfluidic system. After drying under a nitrogen stream, the results were examined as images in gray scale by BIE. Binding of the TM allergen was found to result in an increase of the gray scale value for each appropriate unit.

Sensitivity and Standard Curve. To verify the sensitivity of BIE for the detection of TM allergens, five levels of serial dilution containing $1,3.125,6.25,12.5$, and $25 \mathrm{mg} \mathrm{L}^{-1}$ of the shrimp TM allergen protein were prepared in PBST. A quantitative analysis of the TM allergen was performed in advance under the Bradford method. The concentration gradient for the TM allergen was then acquired through collecting measurement of different gray scale values obtained by BIE. These measurements were obtained with four replicates of gradient detection. The concentration gradient of the TM allergen representing the $X$-axis and the gray scale value (acquired by BIE) representing the $Y$-axis were used to calculate the standard curve.

TM Allergen Detection of Commercial Samples. In order to ensure the integrity of our analysis, fresh live shrimp and crab were used as the positive control. Conversely, powdered milk, soy, eggs, fish, and graham bread were selected as negative samples. Processed food such as shrimp crackers, shrimp paste, shrimp meat balls, and shrimp strips were used to detect the TM allergen. TM allergens from commercial samples and proteins from negative samples were extracted by previously described methods. The extracts were then diluted at a ratio of 1:10 with PBST and finally detected and analyzed with four replicates by BIE. 


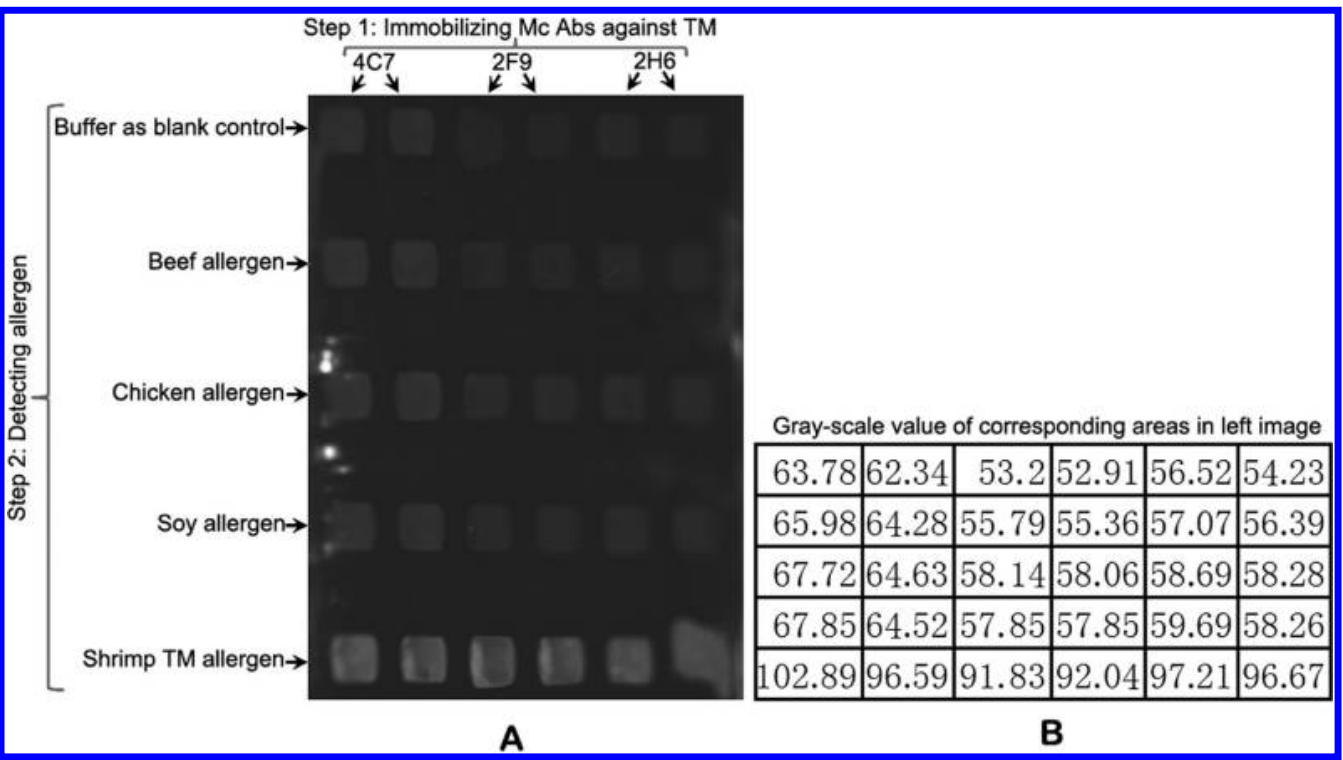

Figure 1. Specificity interaction between shrimp TM allergen and Mc Abs against TM. (A) Image in gray scale. (B) Average value in gray scale of image depicted in A. Small white spots depict in the image represent contaminate spots.

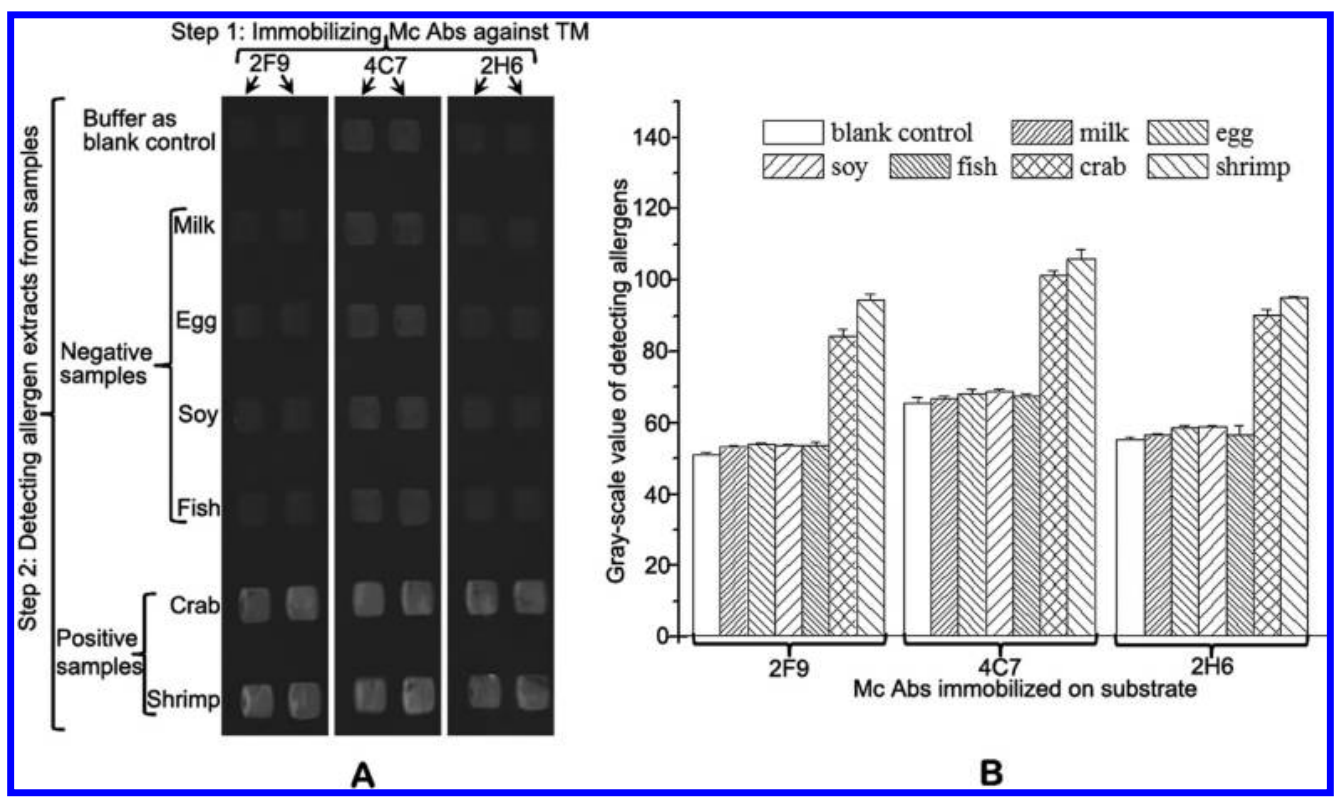

Figure 2. Qualitative detection of the TM allergen using BIE. (A) Image in gray scale of the different food allergens detected. (B) Average gray scale value allergens depicted in A (for two duplicate units).

TM Allergen Detection Using ELISA kit. The commercial ELISA test Shellfish Assay and Fast Crustacean Kits were used for the control method. The protein extraction, detection process, and analysis were executed with three replicates according to the instruction book in ELISA kit.

Statistical Analysis. The gray scale images, gray scale values, and 3-D gray scale distribution map were acquired using the Ellipsometric Imaging Expert System (EIES) of BIE. The results were statistically evaluated by a one-way analysis of variance (AVOVA) using the software Microcal Origin 7.5 (Microcal Software, Inc., Northampton, MA). Statistical differences with P-values under 0.05 were considered significant. $^{33}$

\section{RESULTS AND DISCUSSION}

Specificity Confirmation. In order to verify the specificity of Mc Abs against the TM allergen, we immobilized the beef, chicken, and soy allergens (along with the buffer) on chips to be used as the negative and blank control. The interactions between the shrimp TM allergen and three Mc Abs (2F9, 4C7, and 2H6) were measured with BIE. The results are shown in Figure 1. The shrimp TM allergen was observed to cause a variation in values of the gray scale, while the other allergens did not. This is consistent with the results of the Western blot and ELISA result, ${ }^{30,31}$ and indicates that the shrimp TM allergen interacted with the Mc Abs to become immobilized on substrate (bottom line, Figure 1).

Qualitative Detection of Shrimp and Crab TM Allergen. TM allergen extracts were diluted 10 times prior to use. The signal intensities of allergen extracts from shrimp 


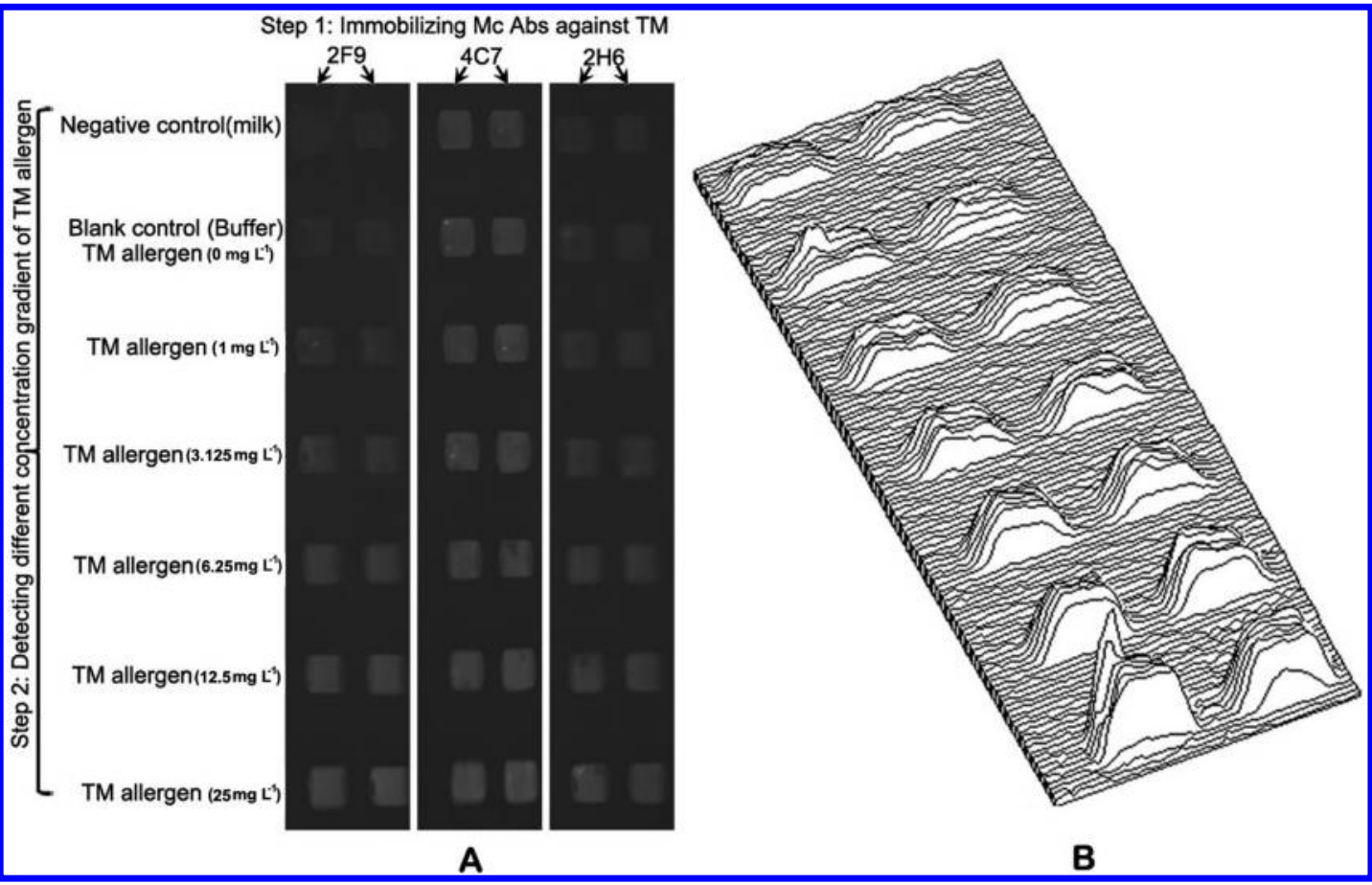

Figure 3. Sensitivity of BIE in the detection of TM, with $\mathrm{Mc} \mathrm{Mbs}(2 \mathrm{~F} 9,4 \mathrm{C} 7$, and $2 \mathrm{H} 6)$ as ligands. (A) Images in gray scale of different TM concentration levels detected. (B) 3-D gray scale distribution map of different concentrations of TM allergen detected, with Mc Mbs $2 \mathrm{H} 6$ representing the ligand portrayed in image $\mathrm{A}$. The same concentration was measured in two duplicate areas.

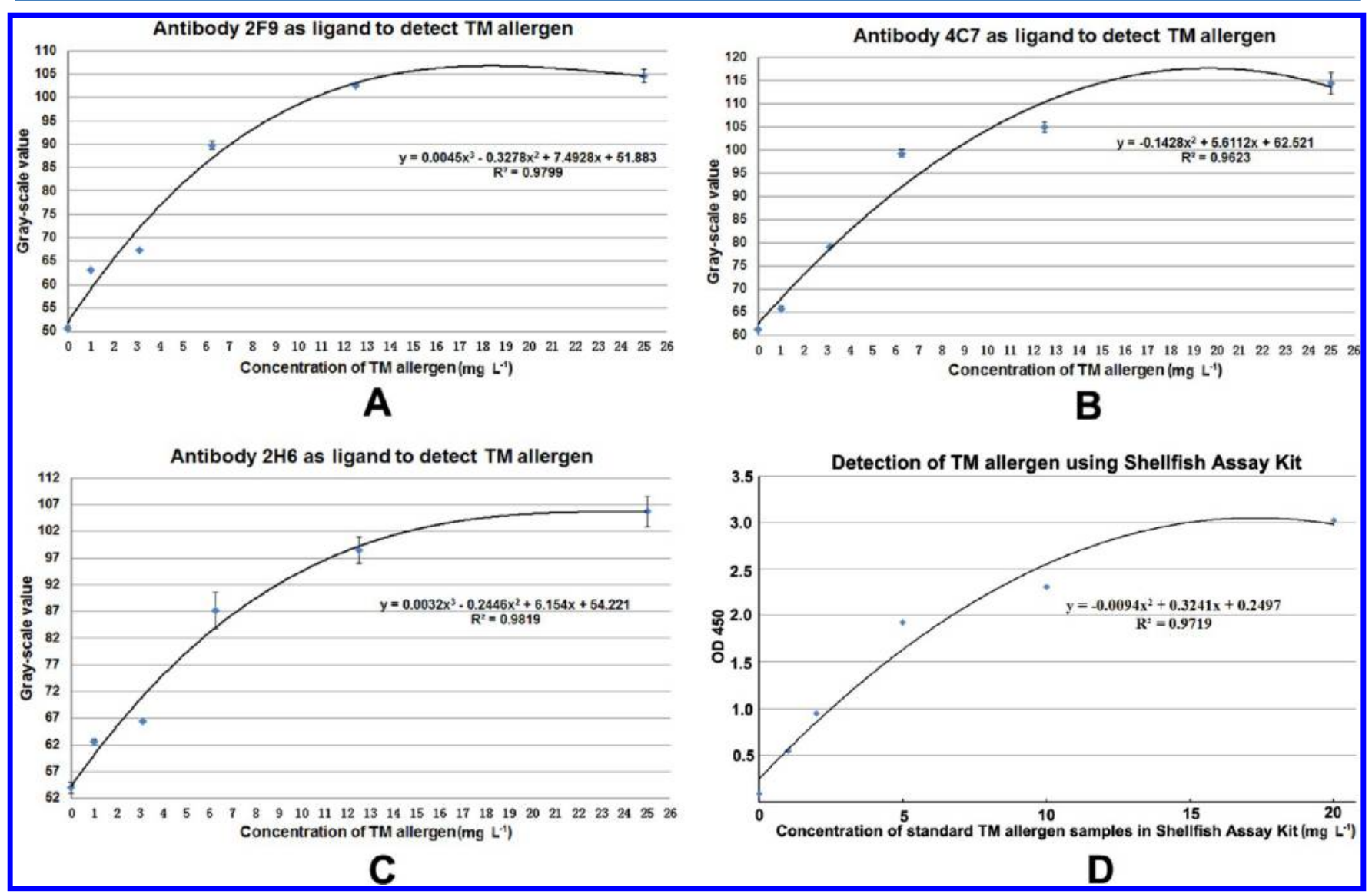

Figure 4. Standard curve of antibodies $2 \mathrm{~F} 9,4 \mathrm{C} 7$, and $2 \mathrm{H} 6$ as ligands to detect TM allergen (five levels of serial dilution containing 1, 3.125, 6.25, 12.5 , and $25 \mathrm{mg} \mathrm{L}^{-1}$ of shrimp TM allergen protein were prepared in PBST). (A) Antibody 2F9. (B) Antibody 4C7. (C) Antibody 2H6. (D) Detection standard TM allergen samples using Shellfish Assay Kit. 


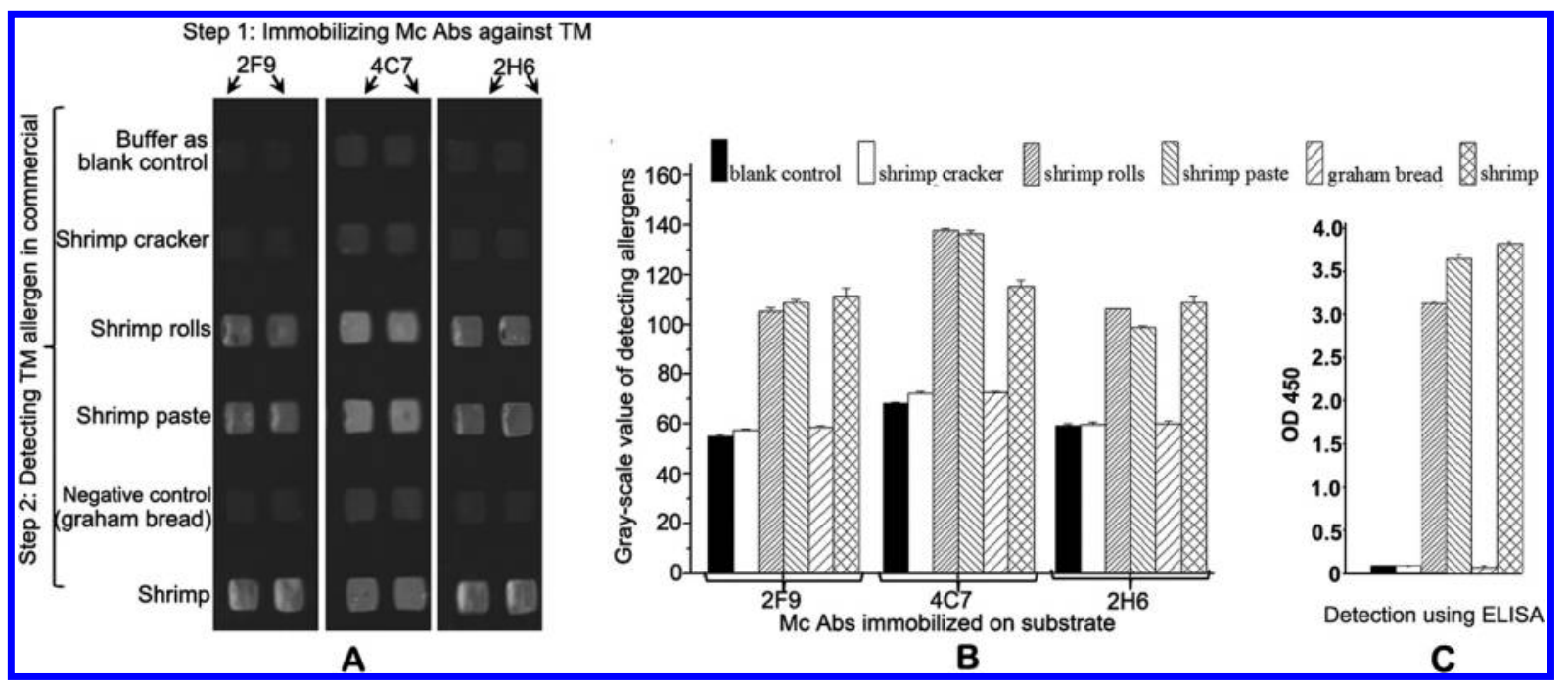

Figure 5. Detection of TM in commercial food products using the BIE. (A) Image in gray scale of TM detected by BIE in commercial food products. (B) Average gray scale values corresponding to the gray scale images (for two duplicate units). (C) Using ELISA as control method to detect commercial samples.

and crab were obviously brighter than that of the blank control and other samples (milk, soy, egg, and fish). These results are shown in Figure 2 and indicate that Mc Abs interacting with TM has a greater affinity for allergen extracts from shrimp and crab than the other food allergens measured. Take antibody 2F9 as an example (Figure $2 \mathrm{~B}$ ). The gray scale value of the blank control was measured at $51.6 \pm 0.5$, and the values of milk, eggs, soy, fish, crab, and shrimp were measured at $53.2 \pm$ $0.4,53.9 \pm 0.7,53.6 \pm 0.4,53.6 \pm 1.2,84.3 \pm 1.9$, and $94.3 \pm$ 1.8 , respectively. The value of shrimp was about $11.9 \%$ higher than that of crab, and the values of shrimp and crab indicate significant reactions in comparison with those of milk, eggs, soy, fish, and the blank control sample (P-value $<0.05$, F-test). The results for antibodies $4 \mathrm{C} 7$ and $2 \mathrm{H} 6$ also showed a similar trend with the values of shrimp again showing a significant difference (P-value $<0.05$, F-test). The results were measured at $105.9 \pm$ 2.9 and $95.1 \pm 0.5$, about $4.4 \%$ and $5.4 \%$ higher than the values measured for crab. However, the interaction of antibody $2 \mathrm{~F} 9$ with the crab allergenic extract was slightly lower than those measured for antibodies 4C7 and 2H6. It was thus concluded that TM has a higher amino acid sequence homology between shrimp and crab, subsequently verifying the reported conclusions. $^{34,35}$ Because of a highly conserved amino acid sequence in TM, the three different antibodies used in our study could potentially be available for direct detection analyses of other crustaceans (such as prawn and lobster).

Sensitivity and Stability of the Detection. Five concentration gradients of the TM standard sample measured in serial diluted samples were applied to the confirmation of the sensitivity (Figure 3). The results show that the change in signal intensity was consistent with the increase in TM concentration. The sensitivity in detecting TM levels for antibodies $2 \mathrm{~F} 9,4 \mathrm{C} 7$, and $2 \mathrm{H} 6$ reached up to $1.0 \mathrm{mg} \mathrm{L}^{-1}$. The gray scale values of the blank control were measured at $56.0 \pm$ 4.6, $70.2 \pm 7.2$, and $57.9 \pm 4.4$, respectively, with the values of the negative control (milk) measured at $56.7 \pm 1.1,70.8 \pm 2.3$, and $58.9 \pm 3.1$, about $1.3 \%, 0.85 \%$, and $0.17 \%$ higher than the values of the blank control. The values of the positive sample $\left(1.0 \mathrm{mg} \mathrm{L}^{-1} \mathrm{TM}\right)$ were measured at $63.1 \pm 0.3,74.5 \pm 7.2$, and $63.6 \pm 3.2$, about $12.6 \%, 6.13 \%$, and $9.84 \%$ higher than that of the blank controls. These values were also measured at about
$11.1 \%, 5.23 \%$, and $7.98 \%$ higher than those of the negative controls, respectively. This indicates that the sensitivity of this assay was $1.0 \mathrm{mg} \mathrm{L}^{-1}$. Currently, there are few existing studies on the lowest dose of shrimp allergens that can elicit a clinical allergic reaction. Reactions with up to 14 and $16 \mathrm{~g}$ of shrimp (equivalent to $32 \mathrm{mg}$ of extracted shrimp protein) have been reported in a double-blind placebo-controlled food challenge (DBPCFC) study. ${ }^{36,37}$ The European Academy of Allergology and Clinical Immunology proposed starting doses of shrimp at $5 \mathrm{mg} .{ }^{38}$ Therefore, the sensitivity of BIE in our study should be sufficient to satisfy requirements for practical use.

Standard Curve. The standard curve of antibodies 2F9, $4 \mathrm{C} 7$, and $2 \mathrm{H} 6$ and the detection of the shrimp TM allergen standard sample are shown in Figure 4. Each variation of gray scale values was linked to a corresponding concentration of the TM allergen in the range of $1-25 \mathrm{mg} \mathrm{L}^{-1}$. TM allergen standard allergen samples $\left(0,5,10,15,20 \mathrm{mg} \mathrm{L}^{-1}\right)$ in the Shellfish Assay Kit were detected by the control detection method. It was concluded that different serial dilutions of samples could be distinguished, thus providing the foundations for a quantitative detection of unknown concentrations of samples in the future. ${ }^{22}$ The calibration curve could be drawn for a quantitative detection of TM hardly recognizable from methods aside from BIE, in which the immunological or genomic properties could only be detected through an additional amplification step. ${ }^{26}$

Detection of TM Allergen in Commercial Samples. In order to confirm the reliability and accuracy of BIE, five different commercial samples were chosen as detected objects (Figure 4). The results of this analysis indicate that the signal intensities of shrimp rolls, shrimp paste, and shrimp were significantly higher than those measured for blank control (PBST) and negative control (graham bread), while the signal intensity measured for the shrimp cracker was nearly equal to those of the control groups (Figure 5A). When compared with the gray scale values of the blank control $(55.2 \pm 0.7)$ and negative control $(58.7 \pm 0.9)$ for antibody $2 \mathrm{~F} 9$ (Figure 5B), the value of shrimp rolls, shrimp paste, and shrimp were measured at $105.3 \pm 1.3,109.0 \pm 0.8$, and $115.2 \pm 3.3$, respectively. These values were also observed to increase significantly (Pvalue $<0.05$, F-test). However, the value of the shrimp cracker 
(with shrimp declared on the label) was measured at only 57.5 \pm 0.4 , which falls between the values measured for the blank and negative control. The detection results for antibodies $4 \mathrm{C} 7$ and $2 \mathrm{H} 6$ were similar (Figure 5B). In order to verify the accuracy of this assay, ELISA test kits were used with the results shown to be consistent with those of the BIE detection (Figure 5C). Detection results of the shrimp cracker and graham bread were similar to those of the blank control $\left(0 \mathrm{mg} \mathrm{L}^{-1} \mathrm{TM}\right.$ allergen standard allergen samples). TM allergen concentrations of other commercial samples were measured at greater than $20 \mathrm{mg} \mathrm{L}^{-1}$.

Failure to detect TM in shrimp cracker could be due to the modification, denaturation, and degradation of the allergen protein in a highly processed food. Our studies confirmed that the results of the BIE detection are consistent with those of the ELISA detection. For the analysis of undestroyed allergens in food, this technique should be considered an effective monitoring and assessment tool to verify food allergen labeling, and also to track the sources of allergen contamination in food.

\section{CONCLUSION}

A label-free and multiplex biosensor has been developed and validated to identify and measure levels of the major tropomyosin allergen present in shrimp and crab. It is feasible to use the biosensor as a qualitative and quantitative highthroughput tool to detect tropomyosin allergen in on-site tests. This method is also shown to be suitable for the analysis and surveillance of different ingredients in processed food and for food allergen labeling. Further, the biosensor may be used to study the structure, function, and allergy mechanisms of food allergens following the future development of real-time biosensors based on imaging ellipsometry.

\section{AUTHOR INFORMATION}

\section{Corresponding Author}

*E-mail: chenyingcaiq@163.com (Y.C.),68511009@163.com (Y.-q.G.).

\section{Present Address}

${ }^{\S}$ Wei Wang's address is National Center of Meat Quality \& Safety Control, Nanjing Agricultural University, China

\section{Author Contributions}

"Wei Wang and Cai Qi have equal contribution to this work.

Notes

The authors declare no competing financial interest.

\section{ACKNOWLEDGMENTS}

We thank Prof. Zhigang Liu (Shenzhen University, China) for his kindly supply of allergen proteins and Mc Abs. The authors acknowledge financial support by the National Natural Science Foundation of the People's Republic of China (31071552), National Basic Research Program (Project 973) of China (2009CB320300) and National Key Technologies R\&D Program of Ministry of Science and Technology of the People's Republic of China (2011BAK10B03), and Basic Scientific Research Special Foundation of Chinese Academy of Inspection and Quarantine (2010JK002).

\section{REFERENCES}

(1) Sziksz, E.; Vannay, A.; Haczku, A. Allergy 2012, 67, 293-295.

(2) Golias, J.; Schwarzer, M.; Wallner, M.; Kverka, M.; Kozakova, H.; Srutkova, D.; Klimesova, K.; Sotkovsky, P.; Palova-Jelinkova, L.; Ferreira, F.; Tuckova, L. PLoS One 2012, 7, e37156.
(3) Paschke, A.; Ulberth, F. Anal. Bioanal. Chem. 2009, 395, 15-16.

(4) Subba Rao, P. V.; Rajagopal, D.; Ganesh, K. A. Allergy 1998, 53, $44-47$.

(5) Shanti, K. N.; Martin, B. M.; Nagpal, S.; Metcalfe, D. D.; Rao, P. V. I. Immunol. 1993, 151, 5354-5363.

(6) Morgan, J. E.; O’Neil, C. E.; Daul, C. B.; Lehrer, S. B. J. Allergy Clin. Immunol. 1989, 83, 1112-1117.

(7) Leung, P. S.; Chen, Y. C.; Mykles, D. L.; Chow, W. K.; Li, C. P.; Chu, K. H. Mol. Mar. Biol. Biotechnol. 1998, 7, 12-20.

(8) Abdel Rahman, A. M.; Lopata, A. L.; O’Hehir, R. E.; Robinson, J. J.; Banoub, J. H.; Helleur, R. J. I. Mass Spectrom. 2010, 45, 372-381.

(9) Zheng, L. N.; Lin, H.; Pawar, R.; Li, Z. X.; Li, M. H. Food Chem. Toxicol. 2011, 49, 2954-2960.

(10) Maier, I.; Morgan, M. R.; Lindner, W.; Pittner, F. Anal. Chem. 2008, 80, 2694-2703.

(11) Crespo, J. F.; Pascual, C.; Helm, R.; Sanchez-Pastor, S.; Ojeda, I.; Romualdo, L.; Martin-Esteban, M.; Ojeda, J. A. Allergy 1995, 50, 918-924.

(12) Julka, S.; Kuppannan, K.; Karnoup, A.; Dielman, D.; Schafer, B.; Young, S. A. Anal. Chem. 2012, 84, 10019-10030.

(13) Eissa, S.; Tlili, C.; L'hocine, L.; Zourob, M. Biosens. Bioelectron. 2012, 38, 308-313.

(14) Jin, G.; Tengvall, P.; Lundstrom, I.; Arwin, H. Anal. Biochem. 1995, 232, 69-72.

(15) Jin, G.; Jansson, R.; Arwin, H. Rev. Sci. Instrum. 1996, 67, 29302936.

(16) Wang, Z. H.; Jin, G. I. Biochem. Biophvs. Methods 2003, 57, $203-$ 211.

(17) Wang, Z. H.; Meng, Y. H.; Ying, P. Q.; Qi, C.; Jin, G. Electrophoresis 2006, 27, 4078-4085.

(18) Chen, Y. Y.; Meng, Y. H.; Jin, G. Appl. Opt. 2007, 46, 84758481.

(19) Arwin, H.; Welinklinstorm, S.; Jansson, R. J. Colloid Interface Sci. 1993, 156, 377-382.

(20) Stenberg, M.; Nygren, H. J. de Phvsiaue 1983, C10, 83-86.

(21) Qi, C.; Zhu, W.; Niu, Y.; Zhang, H. G.; Zhu, G. Y.; Meng, Y. H.; Chen, S.; Jin, G. L. Viral. Hepatitis 2009, 16, 822-832.

(22) Zhang, H. G.; Qi, C.; Wang, Z. H.; Jin, G.; Xiu, R. J. Clin. Chem. 2005, 51, 1038-1040.

(23) Zhang, Y. B.; Chen, Y. Y.; Jin, G. Sens. Actuators, B 2011, 159, $121-125$.

(24) Bae, Y. M.; Park, K. W.; Oh, B. K.; Lee, W. H.; Choi, J. W. Colloids Surf. A 2005, 257, 19-23.

(25) Qi, C.; Tian, X. S.; Chen, S.; Yan, J. H.; Cao, Z.; Tian, K. G.; Gao, G. F.; Jin, G. Biosens. Bioelectron. 2010, 25, 1530-1534.

(26) Qi, C.; Lin, Y.; Feng, J.; Wang, Z. H.; Zhu, C. F.; Meng, Y. H.; Yan, X. Y.; Wan, L. J.; Jin, G. Virus Res. 2009, 140, 79-84.

(27) Qi, C.; Duan, J. Z.; Wang, Z. H.; Chen, Y. Y.; Zhang, P. H.; Zhan, L.; Yan, X. Y.; Cao, W. C.; Jin, G. Biomed. Microdevices 2006, 8, 247-253.

(28) Niu, Y.; Zhuang, J.; Liu, L.; Yan, X. Y.; Jin, G. Thin Solid Films 2011, 519, 2768-2771.

(29) Zhong, X. B.; Reynolds, R.; Kidd, J. R.; Kidd, K. K.; Jenison, R.; Marlar, R. A.; Ward, D. C. Proc. Natl. Acad. Sci. U.S.A. 2003, 100, $11559-11564$.

(30) Chen, H.; Kuang, B. H.; Liu, Z. G.; Huang, Z.; Wu, X. L.; Hu, C.; Zou, J. Immunol. J. 2010, 26, 991-995.

(31) Wu, X. L.; Ji, K. M.; Li, J. N.; Zhan, Z. K.; Liu, Z. G. Food Sci. Technol. 2009, 34, 240-243.

(32) Qiao, B. S. Experimental Technique of Allergy, 2nd ed.; Peking Union Medical College Press: Beijing, 2002.

(33) Freedman, D.; Pisani, R.; Purves, R.; Adhikari, A. Statistics, 2nd ed.; W. W. Norton \& Company: New York, 1991.

(34) Naqpal, S.; Rajappa, L.; Metcalfe, D. D.; Rao, P. V. J. Allergy Clin. Immunol. 1989, 83, 26-36.

(35) Hoffman, D. R.; Collins-Williams, C. J. Allergy Clin. Immunol. 1994, 93, 801-802.

(36) Bernstein, M.; Day, J. H.; Welsh, A. J. Allergy Clin. Immunol. 1982, 70, 205-210. 
(37) Bauermeister, K.; Wangorsch, A.; Garoffo, L. P.; Reuter, A.; Conti, A.; Taylor, S. L.; Lidholm, J.; Dewitt, A. M.; Enrique, E.; Vieths, S.; Holzhauser, T.; Ballmer-Weber, B.; Reese, G. Mol. Immunol. 2011, 48, 1983-1992.

(38) Bindslev-Jensen, C.; Ballmer-Weber, B. K.; Bengtsson, U.; Blanco, C.; Ebner, C.; Hourihane, J.; Knulst, A. C.; Moneret-Vautrin,

D. A.; Nekam, K.; Niggemann, B.; Osterballe, M.; Ortolani, C.; Ring, J.; Schnopp, C.; Werfel, T. Allergy 2004, 59, 690-697. 